\title{
A THEORY OF A GENERAL NET ON A SURFACE*
}

\author{
BY \\ VERNON G. GROVE

\section{InTRODUCTION}

The purpose of this investigation is to carry on the researches of G. M. Green concerning the projective differential geometry of nets of space curves. We assume that the net is not a conjugate net, and, unless otherwise stated, that it is not the asymptotic net. We assume, furthermore, that the sustaining surface is non-developable. The present investigation may, therefore, be considered as an extension of Green's paper on Nets of space curves. $\dagger$

We summarize for future reference some of the results obtained by Green in the paper cited. Let the homogeneous coördinates $y^{(1)}, y^{(2)}, y^{(3)}, y^{(4)}$ of a point in projective space of three dimensions be given as analytic functions

$$
y^{(k)}=y^{(k)}(u, v) \quad(k=1,2,3,4)
$$

of two variables $u$ and $v$. Let $C_{u}$ denote the curve defined by $v=$ const., and $C_{v}$ the curve defined by $u=$ const. The net of curves $C_{u}, C_{v}$ lies on a surface $S_{y}$. If this net is not a conjugate net, the four functions $y^{(k)}(u, v)$ satisfy a system of partial differential equations of the form

$$
\begin{aligned}
& y_{u u}=a y_{u v}+b y_{u}+c y_{v}+d y, \\
& y_{v v}=a^{\prime} y_{u v}+b^{\prime} y_{u}+c^{\prime} y_{v}+d^{\prime} y \quad\left(1-a a^{\prime} \neq 0\right) .
\end{aligned}
$$

The coefficients of system (2) are related by four conditions of complete integrability, one of which $\ddagger$ is

$$
a_{u}^{(12)}+c_{u}^{\prime}=a_{v}^{(21)}+b_{v},
$$

wherein $a^{(21)}$ and ${ }^{(12)}$ are defined by the formulas

$$
\begin{aligned}
& \left(1-a a^{\prime}\right) a^{(21)}=a_{v}+a^{\prime} c+b+a\left(a_{u}^{\prime}+a b^{\prime}+c^{\prime}\right), \\
& \left(1-a a^{\prime}\right) a^{(12)}=a_{u}^{\prime}+a b^{\prime}+c^{\prime}+a^{\prime}\left(a_{v}+a^{\prime} c+b\right) .
\end{aligned}
$$

* Presented to the Society, December 26, 1924; received by the editors in December, 1925.

† G. M. Green, Nets of space curves, these Tr a n s action s, vol. 21 (1920), pp. 207-236. Hereafter referred to as $N$ ets.

$\ddagger$ Nets, p. 212. 
Equation (3) shows the existence of a function $f(u, v)$, such that

$$
f_{u}=a^{(21)}+b, \quad f_{v}=a^{(12)}+c^{\prime} .
$$

In case the parametric net is not asymptotic, so that $a a^{\prime} \neq 0,{ }^{*}$ we find it convenient to write

$$
\begin{aligned}
& \alpha=\frac{1}{a}, \beta=-\frac{b}{a}, \quad \gamma=-\frac{c}{a}, \quad \delta=-\frac{d}{a}, \\
& \alpha^{\prime}=\frac{1}{a^{\prime}}, \quad \beta^{\prime}=-\frac{b^{\prime}}{a^{\prime}}, \quad \gamma^{\prime}=-\frac{c^{\prime}}{a^{\prime}}, \quad \delta^{\prime}=-\frac{d^{\prime}}{a^{\prime}} .
\end{aligned}
$$

Each of the points $\rho$ and $\sigma$ defined by

$$
\rho=y_{u}-\gamma y, \quad \sigma=y_{v}-\beta^{\prime} y
$$

is the focal point of the parametric tangent on which it lies. The line joining the points $\rho$ and $\sigma$ is called the ray of the point $y$, or the parametric ray. The line of intersection of the osculating planes at $y$ of the curves $C_{u}$ and $C_{v}$ is called the axis of the point $y$, or the parametric axis. The harmonic conjugate of $y$ with respect to the focal points of the axis is the point $\tau$ defined by the expression

$$
\tau=y_{u v}-\beta^{\prime} y_{u}-\gamma y_{v}-\frac{1}{2} M y,
$$

where

$$
M=b^{(21)}+c^{(12)}+\gamma a^{(12)}+\beta^{\prime} a^{(21)}-b \beta^{\prime}-c^{\prime} \gamma-a \beta^{\prime 2}-a^{\prime} \gamma^{2}-2 \beta^{\prime} \gamma-\beta_{u}^{\prime}-\gamma_{v},
$$

the functions $b^{(21)}, c^{(12)}$ being defined by the formulas

$$
\begin{aligned}
& \left(1-a a^{\prime}\right) b^{(21)}=b_{v}+b^{\prime} c+a\left(b b^{\prime}+b_{u}^{\prime}+d^{\prime}\right), \\
& \left(1-a a^{\prime}\right) c^{(12)}=c_{u}^{\prime}+b^{\prime} c+a^{\prime}\left(c c^{\prime}+c_{v}+d\right) .
\end{aligned}
$$

\section{The Relation $R$ Between two congruences}

The concept of congruences in relation $R$ with respect to the asymptotic net has been treated by Green. $\dagger$ He pointed $\ddagger$ out that the concept can be also applied to any non-conjugate net. We will discuss that relation with respect to a net which is not conjugate.

Let $R^{(u)}$ be the ruled surface formed by the tangents to the curves $C_{u}$ at the points where they meet the curve $C_{v}$ through $y$. Similarly let $R^{(v)}$

* E. J. Wilczynski, Projective differential geometry of curved surfaces, First Memoir, these Transactions, vol. 8 (1907), p. 243.

† G. M. Green, Memoir on the general theory of surfaces and rectilinear congruences, these Transactions, vol. 20 (1919), p. 86. Hereafter referred to as Surfaces.

$\ddagger$ Surfaces, footnote p. 87 . 
be the ruled surface formed by the tangents to $C_{v}$. Let $r$ be a point on the tangent to $C_{u}$ at $y$, and $s$ be a point on the tangent to $C_{v}$ at $y$. The tangent planes at $r$ and $s$, to $R^{(u)}$ and $R^{(v)}$ respectivcly, intersect in a line $l^{\prime}$ through the point $y$ but not lying in the tangent plane to $S_{\nu}$ at $y$. The line $l$ joining $r$ and $s$ and the line $l^{\prime}$ are said to be in relation $R$. It may readily be verified that the line $l$ joining the points

$$
r=y_{u}-\lambda y, \quad s=y_{v}-\mu y,
$$

and the line $l^{\prime}$ joining $y$ to the point

$$
z=y_{u v}-\mu y_{u}-\lambda y_{v},
$$

where $\lambda$ and $\mu$ are arbitrary functions of $u$ and $v$, are lines in relation $R$. For given functions $\lambda, \mu$, the lines $l$ and $l^{\prime}$ generate congruences $\Gamma$ and $\Gamma^{\prime}$; $\Gamma$ and $\Gamma^{\prime}$ are said to be congruences in relation $R$.

We see, from (6) and (7), that the ray and axis of $y$ are in relation $R$.

\section{The DEvelopables AND FOCAL SURfaces of $\Gamma$ AND $\Gamma^{\prime}$}

To every curve $v=v(u)$ on $S_{\nu}$ there corresponds a ruled surface of $\Gamma$. We proceed to find the differential equation satisfied by $v(u)$ in order that this ruled surface be developable. Any point $P$ on $l$ may be written

$$
P=r+\theta s .
$$

As $y$ moves along $v=v(u), P$ describes a curve. The point $d P / d u$ lies on the tangent to this curve at $P$. We find that

$$
\begin{aligned}
\frac{d P}{d u}= & {\left[a+\theta+\left(1+a^{\prime} \theta\right) \frac{d v}{d u}\right] y_{u v}+\left\{d-\lambda_{u}-\mu_{u} \theta+\left(d^{\prime} \theta-\mu_{v} \theta-\lambda_{v}\right) \frac{d v}{d u}\right.} \\
& \left.+\lambda\left(b-\lambda-\mu \theta+b^{\prime} \theta \frac{d v}{d u}\right)+\mu\left[c+\left(c^{\prime} \theta-\lambda-\mu \theta\right) \frac{d v}{d u}\right]\right\} y+() r+() s,
\end{aligned}
$$

the coefficients of $r$ and $s$ being immaterial. The curve $v=v(u)$ therefore corresponds to a developable of $\Gamma$ if, and only if, the coefficients of $y_{u v}$ and $y$ in (11) vanish. Eliminating $\theta$ from the equations so obtained we find the following differential equation of the curve $v=v(u)$ :

$$
\begin{aligned}
& {[d} \\
& \left.\quad-\lambda_{u}+\lambda(b-\lambda)+c \mu+a \lambda \mu+a \mu_{u}\right] d u^{2} \\
& \quad+\left\{\left[a^{\prime}\left(d-\lambda_{u}\right)+a^{\prime} \lambda(b-\lambda)+a^{\prime} c \mu+\mu_{u}\right]\right. \\
& \left.\quad-\left[a\left(d^{\prime}-\mu_{v}\right)+a \mu\left(c^{\prime}-\mu\right)+a b^{\prime} \lambda+\lambda_{v}\right]\right\} d u d v \\
& \quad-\left[d^{\prime}-\mu_{v}+\mu\left(c^{\prime}-\mu\right)+b^{\prime} \lambda+a^{\prime} \lambda \mu+a^{\prime} \lambda_{v}\right] d v^{2}=0 .
\end{aligned}
$$

The curves (12) will be referred to as the $\Gamma$-curves. 
If we eliminate $d v / d u$ from the coefficients of $y_{u v}$ and $y$ in (11) equated to zero, we find the condition on $\theta$ that $P$ be the point of contact of the line $l$ with the edge of regression of the developable to which it belongs. We find that the focal points on $l$ are defined by the roots of the quadratic

$$
\begin{aligned}
& {\left[d^{\prime}-\mu_{v}+\mu\left(c^{\prime}-\mu\right)+b^{\prime} \lambda+a^{\prime} \lambda \mu+a^{\prime} \mu_{u}\right] \theta^{2}} \\
& \quad+\left\{\left[a\left(d^{\prime}-\mu_{v}\right)+a \mu\left(c^{\prime}-\mu\right)+a b^{\prime} \lambda+\mu_{u}\right]\right. \\
& \left.\quad-\left[a^{\prime}\left(d-\lambda_{u}\right)+a^{\prime} \lambda(b-\lambda)+a^{\prime} c \mu+\lambda_{v}\right]\right\} \theta \\
& \quad-\left[d-\lambda_{u}+\lambda(b-\lambda)+c \mu+a \lambda \mu+a \lambda_{v}\right]=0 .
\end{aligned}
$$

In a similar way we find that the developables of the $\Gamma^{\prime}$ congruence cut $S_{y}$ in the curves whose differential equation is

$$
\begin{aligned}
{\left[c^{(21)}\right.} & \left.-c \mu-\lambda_{u}+\lambda\left(a^{(21)}-a \mu-\lambda\right)\right] d u^{2} \\
& +\left\{\left[c^{(12)}-c^{\prime} \lambda-\lambda_{v}+\lambda\left(a^{(12)}-a^{\prime} \lambda-\mu\right)\right]\right. \\
& \left.-\left[b^{(21)}-b \mu-\mu_{u}+\mu\left(a^{(21)}-a \mu-\lambda\right)\right]\right\} d u d v \\
& -\left[b^{(12)}-b^{\prime} \lambda-\mu_{v}+\mu\left(a^{(12)}-a^{\prime} \lambda-\mu\right)\right] d v^{2}=0 .
\end{aligned}
$$

The curves (14) will be called the $\Gamma^{\prime}$-curves. We find also that the focal points of $l^{\prime}$ are defined by the expressions

$$
z_{1}=z+\varphi_{1} y, \quad z_{2}=z+\varphi_{2} y,
$$

where $\varphi_{1}, \varphi_{2}$ are the roots of the quadratic

$$
\begin{aligned}
\varphi^{2} & +\left[c^{(12)}-c^{\prime} \lambda-\lambda_{v}+b^{(21)}-b \mu-\mu_{u}+\lambda\left(a^{(12)}-a^{\prime} \lambda-\mu\right)+\mu\left(a^{(21)}-a \mu-\lambda\right)\right] \varphi \\
& +\left[\left(b^{(21)}-b \mu-\mu_{u}\right)\left(c^{(12)}-c^{\prime} \lambda-\lambda_{v}\right)-\lambda\left(a^{(21)}-a \mu-\lambda\right)\left(b^{(12)}-b^{\prime} \lambda-\mu_{v}\right)\right. \\
& -\left(c^{(12)}-c \mu-\lambda_{u}\right)\left(b^{(12)}-b^{\prime} \lambda-\mu_{v}\right)-\mu\left(a^{(12)}-a^{\prime} \lambda-\mu\right)\left(c^{(21)}-c \mu-\lambda_{u}\right) \\
& \left.+\lambda\left(a^{(12)}-a^{\prime} \lambda-\mu\right)\left(b^{(21)}-b \mu-\mu_{u}\right)+\mu\left(a^{(21)}-a \mu-\lambda\right)\left(c^{(12)}-c^{\prime} \lambda-\lambda_{v}\right)\right]=0 .
\end{aligned}
$$

The harmonic conjugate of $y$ with respect to $z_{1}$ and $z_{2}$ is defined by the formula.

$$
\begin{aligned}
Z= & z-\frac{1}{2}\left[c^{(12)}-c^{\prime} \lambda-\lambda_{v}+b^{(21)}-b \mu-\mu_{u}\right. \\
& \left.+\lambda\left(a^{(12)}-a^{\prime} \lambda-\mu\right)+\mu\left(a^{(21)}-a \mu-\lambda\right)\right] y .
\end{aligned}
$$

The harmonic invariant of the quadratic (12) and the differential equation of the asymptotic curves*

is

$$
a d u^{2}+2 d u d v+a^{\prime} d v^{2}=0
$$

$$
\mu_{u}-\lambda_{v} .
$$

* Nets, p. 213. 
Hence if with Green* we call those congruences $\Gamma$, whose developables correspond to a conjugate net on $S_{y}$, harmonic to the surface, we see that the $\Gamma$-congruence is harmonic to the surface if and only if the expression (17) vanishes. $\dagger$

Green has called those congruences $\Gamma^{\prime}$ whose developables cut $S_{y}$ in a conjugate net conjugate to the surface. We find that the $\Gamma^{\prime}$ congruence is conjugate to $S_{y}$ if and only if

$$
\frac{\partial}{\partial u}\left(c^{\prime}+a^{\prime} \lambda+\mu\right)=\frac{\partial}{\partial v}(b+a \mu+\lambda) .
$$

\section{SOME GENERAL PROPERTIES OF CONGRUENCES IN RELATION $R$}

To the curves $C_{u}$ and $C_{v}$ there correspond two ruled surfaces $S^{(u)}$ and $S^{(v)}$ of the $\Gamma^{\prime}$ congruence. If $C_{v}$ does not coincide with a $\Gamma^{\prime}$-curve, the plane determined by $l^{\prime}$ and the tangent to $C_{v}$ at $y$ is tangent to $S^{(u)}$ at a definite point $\zeta_{1}$ of $l^{\prime}$. This point must be given by an expression of the form

$$
\zeta_{1}=z+\omega y \text {. }
$$

By imposing the condition that $\zeta_{1 u}$ must lie in this plane, we maydetermine $\omega$. We find that the plane determined by the tangent to $C_{v}$ and $l^{\prime}$ touches $S^{(u)}$ in the point

$$
\zeta_{1}=z-\left[b^{(21)}-b \mu-\mu_{u}+\mu\left(a^{(21)}-a \mu-\lambda\right)\right] y .
$$

Similarly the plane determined by the tangent to $C_{u}$ at $y$ and $l^{\prime}$ touches $S^{(0)}$ at the point

$$
\zeta_{2}=z-\left[c^{(12)}-c^{\prime} \lambda-\lambda_{v}+\lambda\left(a^{(12)}-a^{\prime} \lambda-\mu\right)\right] y .
$$

The totality of points $r$ defined by (9) form a surface $S_{r}$. The tangent at $r$ to the curve $u=$ const. on $S_{r}$ meets $l^{\prime}$ in the point

$$
\eta_{1}=z-\left(\lambda_{v}-\lambda \mu\right) y \text {. }
$$

Similarly the tangent at $s$ to the curve $v=$ const. on $S$, meets $l^{\prime}$ in the point

$$
\eta_{2}=z-\left(\mu_{u}-\lambda \mu\right) y \text {. }
$$

The points $\eta_{1}$ and $\eta_{2}$ coincide if and only if $\mu_{u}-\lambda_{v}=0$, that is, if and only if the $\Gamma$ congruence is harmonic to the surface. The points $\zeta_{1}$ and $\zeta_{2}$ coincide if and only if the $\Gamma^{\prime}$ tangents separate the parametric tangents harmonically.

\footnotetext{
* Surfaces, p. 99.

$\dagger$ It is verified readily that, whatever parametric net is used, the line joining $r=y_{u}-\lambda y, s=y_{0}-\mu y$ is harmonic to the surface if $\mu_{4}-\lambda_{0}=0$.
} 


\section{The osculating QUadrics of THE Parametric RULed SURFACES OF TANGENTS}

By methods similar to those used by Green, ${ }^{*}$ we find that the quadrics osculating the parametric ruled surfaces of tangents $R^{(u)}$ and $R^{(v)}$ along their generators through $y$ are respectively

$$
\begin{aligned}
& Q^{(u)}=\frac{1}{2} a^{\prime} x_{3}^{2}-\frac{1}{2} c^{(12)} x_{4}^{2}-x_{1} x_{4}+x_{2} x_{3}+\frac{1}{2}\left(f_{v}-2 c^{\prime}\right) x_{3} x_{4}=0, \\
& Q^{(v)}=\frac{1}{2} a x_{2}^{2}-\frac{1}{2} b^{(21)} x_{4}^{2}-x_{1} x_{4}+x_{2} x_{3}+\frac{1}{2}\left(f_{u}-2 b\right) x_{2} x_{4}=0,
\end{aligned}
$$

the tetrahedron of reference being $y, y_{u}, y_{v}, y_{u v}$.

By comparing $Q^{(u)}$ and $Q^{(v)}$, we find that $Q^{(u)}$ and $Q^{(v)}$ coincide if and only if $a=a^{\prime}=0$, that is, if and only if $C_{u}$ and $C_{v}$ are asymptotic curves. Since the coördinates of $\rho$ defined by $(6)$ are $(\gamma,-1,0,0)$, we see that $\rho$ lies on $Q^{(u)}$, but never on $Q^{(v)}$; similarly $\sigma$ lies on $Q^{(v)}$ but never on $Q^{(u)}$. The polar plane of $\tau$, defined by (7), with respect to $Q^{(u)}$ passes through $\rho$ and will pass through $\sigma$ if and only if the invariant

$$
\mathfrak{B}=a^{\prime} \gamma-\frac{1}{2}\left(f_{v}-2 c^{\prime}\right)
$$

vanishes. The polar plane of $\tau$ with respect to $Q^{(v)}$ passes through $\sigma$ and will pass through $\rho$ if and only if the invariant

$$
\mathfrak{C}^{\prime}=a \beta^{\prime}-\frac{1}{2}\left(f_{u}-2 b\right)
$$

vanishes.

The line $l^{\prime}$ cuts $Q^{(u)}$ and $Q^{(v)}$ in $y$ and the points $Z^{(u)}$ and $Z^{(v)}$ respectively, where

$$
\begin{aligned}
& Z^{(u)}=z+\frac{1}{2}\left[2 \lambda \mu-\lambda\left(a^{(12)}-c^{\prime}-a^{\prime} \lambda\right)+c^{(12)}\right] y, \\
& Z^{(v)}=z+\frac{1}{2}\left[2 \lambda \mu-\mu\left(a^{(21)}-b-a \mu\right)+b^{(21)}\right] y .
\end{aligned}
$$

The point $Z^{(u)}$ is the harmonic conjugate of $y$ with respect to $\eta_{1}$, and $\zeta_{2}$ defined by (21) and $(20) ; Z^{(v)}$ is the harmonic conjugate of $y$ with respect to $\eta_{2}$ and $\zeta_{1}$ defined by (22) and (19).

\section{The $R$-RECIPROCAL CONGRUENCES}

The line $l$ intersects $Q^{(u)}$ in the points $r$ and $P^{(u)}$ defined by

$$
P^{(u)}=a^{\prime} r-2 s
$$

and intersects $Q^{(v)}$ in $s$ and $P^{(v)}$,

$$
P^{(v)}=a s-2 r .
$$

The equations of the tangent planes to $Q^{(u)}$ at $r$ and $P^{(u)}$ are respectively

$$
x_{3}+\lambda x_{4}=0, \quad 2 x_{2}+a^{\prime} x_{3}+2\left(\mu-a^{\prime} \lambda+f_{v}-2 c^{\prime}\right) x_{4}=0 .
$$

\footnotetext{
- Surfaces, pp. 96, 97.
} 
The points $y$ and $\left(0, \mu+\frac{1}{2} f_{v}-c^{\prime}-a^{\prime} \lambda, \lambda,-1\right)$ lie on the intersection of these planes. Therefore the polar line $l^{(u)}$ of $l$ with respect to $Q^{(u)}$ joins $y$ to the point defined by

$$
\zeta^{(u)}=y_{u v}-\left(\mu-\frac{1}{2} f_{v}-c^{\prime}-a^{\prime} \lambda\right) y_{u}-\lambda y_{v} .
$$

Similarly, the polar line $l^{(v)}$ of $l$ with respect to $Q^{(v)}$ joins $y$ to the point

$$
\zeta^{(v)}=y_{u v}-\mu y_{u}-\left(\lambda+\frac{1}{2} f_{u}-b-a \mu\right) y_{v} .
$$

The lines $l^{(u)}$ and $l^{(v)}$ coincide if and only if

$$
\lambda=\frac{1}{2 a^{\prime}}\left(f_{v}-2 c^{\prime}\right), \quad \mu=\frac{1}{2 a}\left(f_{u}-2 b\right) .
$$

Hence the line $l$ joining

$$
r=y_{u}-\frac{1}{2 a^{\prime}}\left(f_{v}-2 c^{\prime}\right) y, \quad s=y_{v}-\frac{1}{2 a}\left(f_{u}-2 b\right) y
$$

has coincident reciprocal polars with respect to $Q^{(u)}$ and $Q^{(v)}$; this reciprocal polar joins $y$ to the point $Z$ defined by

$$
Z=y_{u v}-\frac{1}{2 a}\left(f_{u}-2 b\right) y_{u}-\frac{1}{2 a^{\prime}}\left(f_{v}-2 c^{\prime}\right) y_{v}
$$

Evidently this unique pair of reciprocal polars are in relation $R$; we will call them the $R$-reciprocal lines, and the congruences generated by them the $R$-reciprocal congruences.

It follows from (28) and (6) that $r$ will coincide with $\rho$ if and only if $\mathfrak{B}=0$; and $s$ will coincide with $\sigma$ if and only if $\mathbb{S}^{\prime}=0$. Hence the $R$-reciprocal congruences coincide with the ray and axis congruences if and only if $\mathfrak{B}=\mathfrak{S}^{\prime}=0$.

The quadrics $Q^{(u)}$ and $Q^{(v)}$ intersect in a nodal quartic with node at $y$. The projecting cone of this quartic from $y$ is a quadric cone $K$. As $l^{\prime}$ generates $K$, the line $l$ in relation $R$ to $l^{\prime}$ envelops a conic $C$. The polar line of $y$ with respect to $C$ is the line joining $r s$. The polar planes of the focal points $\rho$ and $\sigma$ with respect to $K$ intersect in the line $y Z$. If $K$ degenerates into two distinct planes, the line $y Z$ is their intersection. In this case $C$ degenerates into the line rs counted twice.

\section{The associate conjugate net}

The net defined by the differential equation

$$
a d u^{2}-a^{\prime} d v^{2}=0
$$

has been called the associate conjugate net.* The tangents at $y$ to the curves 
of this net are the double rays of the involution determined by the parametric tangents and the asymptotic tangents.

Let us call the line joining the focal points of the associate conjugate tangents the associate ray, and the line of intersection of the osculating planes at $y$ of the associate conjugate curves the associate axis. Any point on the tangent to the curve defined by $d v / d u=m, m=\sqrt{a / a^{\prime}}$ is defined by an expression of ti.e form

$$
\bar{\rho}=y_{u}+m y_{v}+\omega_{1} y .
$$

By methods similar to those used in $\S 3$, we determine $\omega_{1}$ so that $\bar{\rho}$ is the focal point of the tangent on which it lies. We obtain

$$
\omega_{1}=\frac{\left(1+a^{\prime} m\right)\left(c+m_{u}-b m\right)-(a+m)\left(c^{\prime} m+m_{v}-b^{\prime} m^{2}\right)}{a+2 m+a^{\prime} m^{2}} .
$$

By changing the sign of $m$ in (31) and (32), we find that the focal point of the tangent to the curve defined by $d v / d u=-m$ is the point

$$
\bar{\sigma}=y_{u}-m y_{v}+\omega_{2} y,
$$

where

$$
\omega_{2}=\frac{\left(1-a^{\prime} m\right)\left(c-m_{u}+b m\right)+(a-m)\left(c^{\prime} m+m_{v}+b^{\prime} m^{2}\right)}{a-2 m+a^{\prime} m^{2}} .
$$

It may be verified from (31) and (33) that the associate ray intersects the parametric tangents in the points

$$
\begin{aligned}
& r=y_{u}-\frac{1}{2}\left(b-b^{\prime} m^{2}-\frac{m_{u}}{m}\right) y, \\
& s=y_{v}-\frac{1}{2}\left(c^{\prime}-c m^{\prime 2}-\frac{m_{v}^{\prime}}{m^{\prime}}\right) y, \quad m m^{\prime}=1 .
\end{aligned}
$$

By comparing (6) with (35), we find that $r$ will coincide with $\rho$ if and only if the invariant

$$
\subseteq=b-b^{\prime} m^{2}-2 \gamma-\frac{m_{u}}{m}
$$

vanishes. Similarly $s$ will coincide with $\sigma$ if and only if the invariant

$$
\mathfrak{S}^{\prime}=c^{\prime}-c m^{\prime 2}-2 \beta^{\prime}-\frac{m_{v}^{\prime}}{m^{\prime}}
$$


vanishes. By comparing (6) and (28), we find that the associate ray coincides with the line $r s$ if and only if the invariants

$$
a^{\prime} \mathfrak{S}+2 \mathfrak{B}, \quad a \mathfrak{S}^{\prime}+2 \mathfrak{S}^{\prime}
$$

vanish.

The associate axis joins $y$ to a point defined by an expression of the form (39)

$$
s=y_{u v}-\mu y_{u}-\lambda y_{v} .
$$

Consider first the curve defined by $d v / d u=m$. The osculating plane at $y$ to this curve is determined by the points $y$

$$
\begin{aligned}
\frac{d y}{d u}= & y_{u}+m y_{v}, \\
\frac{d^{2} y}{d u^{2}}= & \left(a+2 m+a^{\prime} m^{2}\right) y_{u v} \\
& +\left(b+b^{\prime} m^{2}\right) y_{u}+\left(c+m_{u}+c^{\prime} m^{2}+m m_{v}\right) y_{v}+() y,
\end{aligned}
$$

the coefficient of $y$ being immaterial. If we impose the condition that $y z$ shall lie in this plane, we find that $\lambda$ and $\mu$ satisfy the equation

$$
(\lambda-m \mu)\left(a+2 m+a^{\prime} m^{2}\right)=m\left(b+b^{\prime} m^{2}\right)-c-c^{\prime} m^{2}-m m_{v}-m_{\mathbf{u}} .
$$

By changing the sign of $m$ in (40), we find the condition that $y z$ shall lie in the osculating plane at $y$ to the curve defined by $d v / d u=-n$ is

$$
(\lambda+m \mu)\left(a-2 m+a^{\prime} m^{2}\right)=-m\left(b+b^{\prime} m^{2}\right)-c-c^{\prime} m^{2}-m m_{\mathrm{v}}+m_{\mathrm{u}} \text {. }
$$

Solving (40) and (41) for $\lambda$ and $\mu$, we find that the associate axis joins $y$ to the point

$$
z=y_{u v}-\mu y_{u}-\lambda y_{v}
$$

where

$$
\begin{aligned}
& \lambda=\frac{1}{2\left(1-a a^{\prime}\right)}\left(b+b^{\prime} m^{2}+a c^{\prime}+a^{\prime} c-a \frac{m_{v}^{\prime}}{m^{\prime}}+\frac{m_{u}^{\prime}}{m^{\prime}}\right), \\
& \mu=\frac{1}{2\left(1-a a^{\prime}\right)}\left(c^{\prime}+c m^{\prime 2}+a^{\prime} b+a b^{\prime}-a^{\prime} \frac{m_{u}}{m}+\frac{m_{v}}{m}\right) .
\end{aligned}
$$

Equations (7) and (42) show that the associate axis lies in the osculating plane to $C_{v}$ if and only if the invariant

$$
\mathfrak{S}=c^{\prime}+c m^{\prime 2}+a^{\prime} b-a b^{\prime}-2 \beta^{\prime}-a^{\prime} \frac{m_{u}}{m}+\frac{m_{v}}{m}=a^{\prime} \subseteq+\mathfrak{S}^{\prime}
$$


vanishes; it lies in the osculating plane to $C_{u}$ if and only if the invariant

$$
\mathfrak{B}^{\prime}=b+b^{\prime} m^{2}+a c^{\prime}-a^{\prime} c-2 \gamma-a \frac{m_{v}{ }^{\prime}}{m^{\prime}}+\frac{m_{u}{ }^{\prime}}{m^{\prime}}=a \Im^{\prime}+\subseteq
$$

vanishes. From (43) and (44) it follows that the associate ray congruence coincides with the parametric ray congruence if, and only if, the associate axis congruence coincides with the parametric axis congruence.

An interesting special case of this theorem arises in case the parametric tangents form a constant cross ratio with the asymptotic tangents. We may readily verify that the cross ratio formed by these tangents is constant if $a a^{\prime}=$ const. Using the formulas for $\left\{\alpha^{\prime}\right\}$ and $\left\{\beta^{\prime}\right\}$ derived by Green, ${ }^{*}$ we find, except for a factor, that

$$
\begin{aligned}
\left\{\alpha^{\prime}\right\} & =a^{\prime}\left[a a^{\prime} \mathfrak{B}^{\prime}-\frac{1}{2} \frac{\partial}{\partial u}\left(a a^{\prime}\right)\right]-\left(1-\sqrt{1-a a^{\prime}}\right)\left[a a^{\prime} \mathfrak{S}-\frac{1}{2} \frac{\partial}{\partial v}\left(a a^{\prime}\right)\right], \\
\{\beta\} & =a\left[a a^{\prime} \mathfrak{S}-\frac{1}{2} \frac{\partial}{\partial v}\left(a a^{\prime}\right)\right]-(1-\sqrt{1-a a})\left[a a^{\prime} \mathfrak{B}^{\prime}-\frac{1}{2} \frac{\partial}{\partial u}\left(a a^{\prime}\right)\right] .
\end{aligned}
$$

The surface $S_{y}$ is therefore a quadric if, and only if,

$$
a a^{\prime} \mathfrak{B}^{\prime}-\frac{1}{2} \frac{\partial}{\partial u}\left(a a^{\prime}\right)=0, \quad a a^{\prime} \mathfrak{C}-\frac{1}{2} \frac{\partial}{\partial v}\left(a a^{\prime}\right)=0 .
$$

In view of a theorem due to Green, $\dagger$ we may state that for any net, conjugate or not-conjugate, whose tangents form with the asymptotic tangents a constant cross ratio, the associate axis coincides with the axis of the net if and only if the surface is a quadric.

Comparing equations (35) and (39), we find that the associate ray and associate axis are in relation $R$ if and only if $\mathfrak{B}^{\prime}=\sqrt{ }=0$, that is, if and only if they coincide resfectively with the parametric ray and axis. They will be the $R$-reciprocal lines if and only if in addition the invariants (38) vanish. Under these conditions, we find that $a a^{\prime}=$ const. and the invariants (46) vanish. Hence the associate ray and associate axis congruences are the $R$-reciprocal congruences if and only if the sustaining surface is a quadric, and the parameter tangents form a constant cross ratio with the asymptotic tangents.

* G. M. Green, On the theory of curved surfaces and canonical systems in projective differential geometry, these Transactions, vol. 16 (1915), p. 5, formulas (7).

† G. M. Green, Projective differential geometry of one-parameter families of space curves, Second Memoir, American Journal of Mathematics, vol. 38 (1916), p. 317. 


\section{The INVERTED ASSOCIATE NET}

We shall call the net whose differential equation is

$$
a d u^{2}+a^{\prime} d v^{2}=0,
$$

the inverted associate net. The tangents to the curves of this net are the double rays of the involution determined by the parametric and the associate conjugate tangents. We shall call the line joining the focal points of the tangents at $y$ to the inverted associate curves the inverted ray. By changing $m$ to $n=1 / n^{\prime}=\sqrt{-a / a^{\prime}}$ in (31), (32), (33), and (34), we find that the inverted ray cuts the parametric tangents in the points

$$
\begin{aligned}
& r=y_{u}-\frac{1}{2}\left(b-a^{\prime} c+a c^{\prime}-b^{\prime} n^{2}+\frac{n_{u}^{\prime}}{n^{\prime}}-a \frac{n_{v}{ }^{\prime}}{n^{\prime}}\right) y, \\
& s=y_{v}-\frac{1}{2}\left(c^{\prime}-a b^{\prime}+a^{\prime} b-c n^{\prime 2}+\frac{n_{v}}{n}-a^{\prime} \frac{n_{u}}{n}\right) y .
\end{aligned}
$$

Comparing (6) and (48), we find that $r$ will coincide with $\rho$ if and only if $\mathfrak{B}^{\prime}$ vanishes, and $s$ will coincide with $\sigma$ if and only if $\mathfrak{C}$ vanishes.

The line of intersection of the osculating planes at $y$ to the inverted associate curves will be called the inverted axis. By changing $m$ to $n$ in (40) and (41), we find that the inverted axis joins $y$ to the point

$$
z=y_{u v}-\frac{1}{2}\left(c n^{\prime 2}+c^{\prime}-\frac{n_{v}{ }^{\prime}}{n^{\prime}}\right) y_{u}-\frac{1}{2}\left(b^{\prime} n^{2}+b-\frac{n_{u}}{n}\right) y_{v} .
$$

From equations (8) and (50) we find that the inverted associate axis lies in the osculating plane to $C_{v}$ if and only if the invariant $\mathbb{S}^{\prime}$ vanishes; it lies in the osculating plane to $C_{u}$ if and only if $\mathfrak{S}$ vanishes. We note, from (35) and (48), that the associate ray and inverted axis are in relation $R$. They will be $R$-reciprocal lines if, and only if, the invariants (38) vanish.

We may state the results of this section as follows: The inverted axis lies in the osculating plane of a parametric curve if and only if the associate ray meets the tangent to that curve in the focal point. If the associate ray congruence coincides wrth the parametric ray congruence then the inverted axis congruence coincides with the parametric axis congruence and conversely. If any two of the three rays (axes) coincide then all three rays (axes) coincide and all three axes (rays) coincide. If any two of the three ray (axis) congruences coincide with the plane (space) component of the R-reciprocal congruences, then the surface is a quadric, and the parametric tangents form a constant cross ratio with the asymptotic tangents.

Michigan State College, East Lansing, Mich. 